\title{
Evaluation Of Electric Potential Distribution Near FEG-Emitter By Electron Holograpy
}

Tetsuo Oikawa ${ }^{*, * *}$, Takeshi Tomita ${ }^{* *}$, Hyun Soon Park ${ }^{* * *}$ and Daisuke Shindo ${ }^{* * *}$

* Australian Key Center for Microscopy \& Microanalysis, The University of Sydney, Sydney, NSW 2006, Australia

** JEOL Ltd., 1-2 Musashino 3-chome, Akishima, Tokyo 196-8558, Japan

*** Institute of Multidisciplinary Research for Advanced Materials, Tohoku University, 2-1-1 Katahira, Aoba-ku, Sendai 980-8077, Japan

Electric potential distribution near a field emission gun (FEG)-emitter was visualized by electron holography in order to evaluate a performance of the FEG, which is now used in transmission electron microscope (TEM) widely. In this experiment, an FEG-emitter was set at a specimen holder together with an electrode facing it. Electron holograms were recorded with applying the voltages between the emitter and the electrode; thus, the field distribution was observed successfully and the technique has been established for FEG-emitter evaluation.

It is very important and interesting to observe electric potential distribution near an FEG-emitter in order to evaluate a performance and a quality of the FEG, which is now widely used in both transmission and scanning electron microscopes. This paper reports the results of experiments performed by an electron holography technique combined with a newly developed specimen holder, which enables us to set an FEG-emitter and an electrode as cathode and anode, respectively.

Figure 1 shows schematic illustrations of the specimen holder and the instruments used in this experiment. The newly developed holder was installed into a $J E M-3000 F ; 300 \mathrm{kV}$ FE-TEM, and electron holograms were recorded under the condition of applying the voltages between the emitter and the electrode. A distance between the $\mathrm{W}-\mathrm{ZrO}_{2}$ emitter and the electrode can be precisely evaluated on the specimen stage [1], whose movement is controlled by a stepping motor and piezo drives. The bias voltages was $40-100 \mathrm{~V}$, so that they correspond to the electric field of $0.02-0.05 \mathrm{~V} / \mathrm{nm}$.

Figure 2 shows TEM images of a $\mathrm{W}-\mathrm{ZrO}_{2}$ emitter, and electric potential distribution observed at the bias voltage of $100 \mathrm{~V}$. The bias voltage is applied to the electrode having higher electric potential over the W$\mathrm{ZrO}_{2}$ emitter. It was observed that a surface of tungsten (100) at the top of the emitter was clean and flat as shown in Fig. 2(a). Further, the electric potential distribution (Fig. 2 (c)) extending from the surface of the emitter to vacuum was observed clearly by phase-reconstruction from the hologram (Fig. 2 (b)).

The specimen holder developed in this experiment allows us not only to apply voltage [2] but also to apply mechanical stimulation for reconstruction of the emitter surface, by the piezo-drive. The technique developed in this experiment is expected to apply to the evaluation of the FEG and in situ observation of electric potential distribution of other emitters.

[1] Y. Naitoh et al., Surface Science, 433-435 (1999) 627.

[2] J. Cumings et al., Phys. Rev. Lett., 88 (2002) 056804. 
(a)

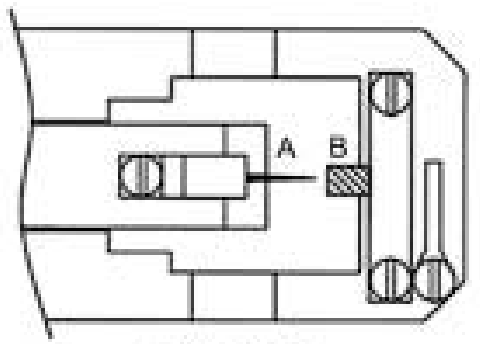

$A: W-\mathrm{ZrO}_{2}$ lip

B: Electrode

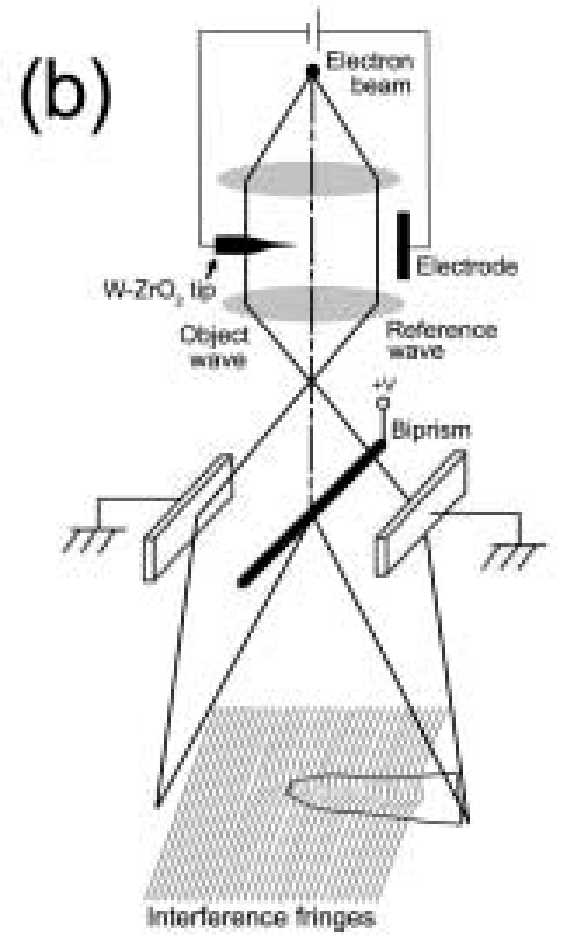

Fig. 1 (a) Schematic illustration of a part of a specimen holder equipped with a $\mathrm{W}-\mathrm{ZrO}_{2}$ emitter. (b) Schematic illustration of the instruments used in this experiment. A W- $\mathrm{ZrO}_{2}$ emitter is set at a specimen holder together with an electrode face to it.
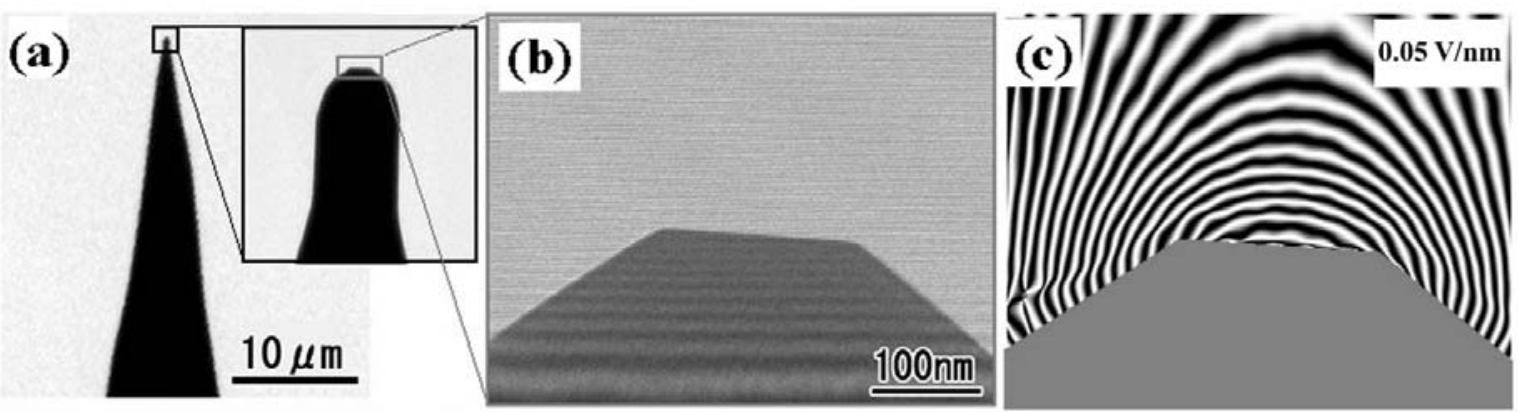

Fig. 2 (a) TEM images of a $\mathrm{W}-\mathrm{ZrO}_{2}$ emitter.

(b) An electron hologram observed at a bias voltage of $100 \mathrm{~V}$.

(c) A reconstructed phase image obtained from the hologram of (b), showing electric potential distribution of a $\mathrm{W}-\mathrm{ZrO}_{2}$ emitter. 